\title{
Association of Preterm Birth with Depression and Particulate Matter: Machine Learning Analysis Using National Health Insurance Data
}

\author{
Kwang-Sig Lee ${ }^{1}$, Hae-In Kim ${ }^{1,2,3}$, Ho Yeon Kim ${ }^{4}{ }^{\circledR}$, Geum Joon Cho ${ }^{5}$, Soon Cheol Hong ${ }^{3}$, Min Jeong Oh ${ }^{5}$, \\ Hai Joong Kim ${ }^{4}$ and Ki Hoon Ahn ${ }^{3, *(1)}$
}

check for

updates

Citation: Lee, K.-S.; Kim, H.-I.; Kim, H.Y.; Cho, G.J.; Hong, S.C.; Oh, M.J.; Kim, H.J.; Ahn, K.H. Association of Preterm Birth with Depression and Particulate Matter: Machine Learning Analysis Using National Health Insurance Data. Diagnostics 2021, 11, 555. https://doi.org/10.3390/ diagnostics 11030555

Academic Editor: Stefano Guerriero

Received: 8 January 2021

Accepted: 18 March 2021

Published: 19 March 2021

Publisher's Note: MDPI stays neutral with regard to jurisdictional claims in published maps and institutional affiliations.

Copyright: (c) 2021 by the authors. Licensee MDPI, Basel, Switzerland. This article is an open access article distributed under the terms and conditions of the Creative Commons Attribution (CC BY) license (https:// creativecommons.org/licenses/by/ $4.0 /)$.
1 AI Center, Korea University Anam Hospital, Seoul 02841, Korea; ecophy@hanmail.net (K.-S.L.); radis13@korea.ac.kr (H.-I.K.)

2 School of Industrial Management Engineering, Korea University, Seoul 02841, Korea

3 Department of Obstetrics \& Gynecology, Korea University Anam Hospital, Seoul 02841, Korea; novak082@korea.ac.kr

4 Department of Obstetrics \& Gynecology, Korea University Ansan Hospital, Ansan 15355, Korea; shinbi7873@gmail.com (H.Y.K.); haijkim@korea.ac.kr (H.J.K.)

5 Department of Obstetrics \& Gynecology, Korea University Guro Hospital, Seoul 08308, Korea; md_cho@hanmail.net (G.J.C.); mjohmd@korea.ac.kr (M.J.O.)

* Correspondence: akh1220@hanmail.net; Tel.: +82-02-920-6777

\begin{abstract}
This study uses machine learning and population data to analyze major determinants of preterm birth including depression and particulate matter. Retrospective cohort data came from Korea National Health Insurance Service claims data for 405,586 women who were aged 25-40 years and gave births for the first time after a singleton pregnancy during 2015-2017. The dependent variable was preterm birth during 2015-2017 and 90 independent variables were included (demographic/socioeconomic information, particulate matter, disease information, medication history, obstetric information). Random forest variable importance was used to identify major determinants of preterm birth including depression and particulate matter. Based on random forest variable importance, the top 40 determinants of preterm birth during 2015-2017 included socioeconomic status, age, proton pump inhibitor, benzodiazepine, tricyclic antidepressant, sleeping pills, progesterone, gastroesophageal reflux disease (GERD) for the years 2002-2014, particulate matter for the months January-December 2014, region, myoma uteri, diabetes for the years 2013-2014 and depression for the years 2011-2014. In conclusion, preterm birth has strong associations with depression and particulate matter. What is really needed for effective prenatal care is strong intervention for particulate matters together with active counseling and medication for common depressive symptoms (neglected by pregnant women).
\end{abstract}

Keywords: preterm birth; particulate matter; depression

\section{Introduction}

Preterm birth is a major part of disease burden for newborns and children on the globe [1-4]. Every year 15 million babies are born preterm in the world and preterm birth is a main contributor for global neonatal and childhood mortality, i.e., 1 million deaths among those aged $0-4$ years [1,2]. For example, one out of every 10 babies was preterm in the United States during 2003-2012, that is, 5,042,982 (12.2\%) of 41,206,315 newborns [3]. Indeed, cost-effective interventions are expected to prevent three quarters of mortality from preterm birth [4]. A recent review reports that the following maternal variables are important predictors of preterm birth: demographic/socioeconomic determinants (age, below high school graduation, urban region, insurance, marriage, religion), disease information (delivery/pregestational body mass index, predelivery systolic/diastolic blood pressure, upper gastrointestinal tract symptom, gastroesophageal reflux disease, Helicobacter pylori, 
gestational diabetes mellitus, systemic lupus erythematosus, increased cerebrospinal fluid and reduced cortical folding due to impaired brain growth), medication history (progesterone, calcium channel blocker, hydroxychloroquine sulfate) and obstetric information (parity, twins, infant sex, prior preterm birth, prior cone biopsy, cervical length, myomas and adenomyosis) [5].

Moreover, emerging literature requests due attention to the significant effects of depression and air pollution on preterm birth [6-15]. Two systematic reviews reported that prenatal or gestational depression is an important risk factor for preterm birth [6,7]. In addition, two systematic reviews [8,9] and several population-based cohort studies [10-15] confirmed a positive association between air pollution and preterm birth. These populationbased cohort studies covered various areas and periods including the San Joaquin Valley (the United States, 2000-2006) [10], Ohio (the United States, 2007-2010) [11], Utah (the United States, 2002-2010) [12], Ontario (Canada, 2005-2012) [13], Wuhan (China, 20112013) [14] and Korea (2010-2013) [15]. However, the number of predictors in the existing literature has been limited to 14 and no effort has been made based on machine learning in this direction. In this context, this study uses machine learning and population data to analyze major determinants of preterm birth including depression and particulate matter. This study includes a population-based cohort of 405,586 participants and the most comprehensive set of 90 predictors such as demographic/socioeconomic determinants, particulate matter, disease information, medication history and obstetric information.

\section{Materials and Methods}

\subsection{Participants}

Retrospective cohort data for this study came from Korea National Health Insurance Service claims data for 405,586 women, aged 25-40 years who gave birth for the first time after a singleton pregnancy during 2015-2017. South Korea runs a compulsory, universal health insurance service program and Korea National Health Insurance Service claims data cover most health events of all citizens residing in Korea (for more details, visit https:/ / www.nhis.or.kr/static/html/wbd/g/a/wbdga0401.html, accessed on 15 March 2021). This retrospective study was approved by the Institutional Review Board (IRB) of Korea University Anam Hospital on 5 November 2018 (2018AN0365). Informed consent was waived by the Institutional Review Board (IRB) given that data were deidentified.

\subsection{Variables}

The dependent variable was preterm labor and birth during 2015-2017 (birth between 20 weeks and 0 day and 36 weeks and 6 days of gestation). Four categories of preterm labor and birth were defined based on ICD-10 Code: (1) PTB 1-preterm birth with premature rupture of membranes (PROM) only; (2) PTB 2-preterm labor and birth without PROM; (3) PTB 3-PTB 1, PTB 2 or both; (4) PTB 4-PTB 3 or other indicated preterm birth (Supplementary Table S1). This variable was coded as "no" vs. "yes". The following 90 independent variables were included: (1) demographic/socioeconomic determinants in 2014 such as age (years), socioeconomic status measured by an insurance fee with the range of 1 (the highest group) to 20 (the lowest group), and region (city) (no vs. yes); (2) particulate matter $\left(\mathrm{PM}_{10}\right)$ for each of the months January-December 2014; (3) disease information (no vs. yes) for each of the years 2002-2014, i.e., depression, diabetes, gastroesophageal reflux disease (GERD), hypertension and periodontitis; (4) medication history (no vs. yes) in 2014, i.e., benzodiazepine, calcium channel blocker, nitrate, progesterone, proton pump inhibitor, sleeping pills and tricyclic antidepressant; (5) obstetric information (no vs. yes) in 2014 such as in vitro fertilization, myoma uteri and prior cone. The 65 disease variables were denoted as Depression_2002, .. , Depression_2014,Diabetes_2002, .. , Diabetes_2014, GERD_2002, ... , GERD_2014,Hypertension_2002, ... , Hypertension_2014, and Periodontitis_2002, ... , Periodontitis_2014. The disease information and the medication history were screened from ICD-10 and ATC codes, respectively (Supplementary Tables S1 and S2). Indeed, diabetes was defined as fasting glucose equal to or higher than $126 \mathrm{mg} / \mathrm{dL}$ or antidiabetic 
medication. Likewise, hypertension was defined as systolic/diastolic blood pressure equal to or higher than $140 / 90 \mathrm{mmHg}$ or antihypertensive medication [16]. Finally, particulate matter was denoted as PM_2014_01 (2014 January), .. , PM_2014_12 (December 2014) and its monthly average at a district level was obtained from [17]. Introducing the disease and particulate matter variables as above (so called "distributed lag variables") is one efficient way to analyze the effects of important independent variables in past periods on the dependent variable in the current period.

\subsection{Analysis}

Logistic regression, the random forest and the artificial neural network were applied and compared for the prediction of preterm birth [18]. Data on 402,092 observations with full information were divided into training and validation sets with a 70:30 ratio (281,464 vs. 120,628 observations). Accuracy, a ratio of correct predictions among 120,628 observations, was introduced as a criterion for validating the models trained. Random forest variable importance, which measures the contribution of a variable for the performance of the model, was used for identifying major determinants of preterm birth and testing its associations with depression, particulate matter and other predictors. R-Studio 1.3.959 (R-Studio Inc., Boston, MA, USA) was employed for the analysis during 1 August 2020-31 December 2020.

\section{Results}

Descriptive statistics for participants' preterm birth and its determinants are shown in Table 1. Among 405,586 participants, 21,732 (5.40\%), 8927 (2.22\%), 27,752 (6.90\%) and 28,845 (7.17\%) belonged to PTB 1, 2, 3 and 4, respectively. The median age and socioeconomic status of the participants were 29 and 12, respectively. Among the participants, 126,008 $(31.34 \%)$ and $63,066(15.68 \%)$ had proton pump inhibitor and tricyclic antidepressant medications in 2014, respectively. The share of those with depression registered a steady growth from $0.18 \%$ in 2002 to $1.36 \%$ in 2014 . The monthly averages of $\mathrm{PM}_{10}$ in Korea's seven metropolitan areas for the year 2014 were 56 (January), 50 (February), 52 (March), 52 (April), 64 (May), 44 (June), 39 (July), 30 (August), 33 (September), 35 (October), 44 (November) and 42 (December) in terms of $10^{-6} \mathrm{~g} / \mathrm{m}^{3}$, respectively. In terms of accuracy, the random forest was similar with logistic regression and the artificial neural network $(94.50 \%, 97.66 \%$, 93.08\% and 92.83\% for PTB 1, PTB 2, PTB 3 and PTB 4 in Table 2, respectively). The results of undersampling are shown in Table 3. Undersampling is an approach to match the sizes of two groups (participants with and without preterm birth) so that the training of machine learning can be balanced between the two groups. Undersampling leads to slight improvement in the performance (the area under the receiver-operating-characteristic curve) of the random forest, e.g., from 0.5585 to 0.5803 in the case of PTB 2.

Table 1. Descriptive statistics on preterm birth and its determinants.

\begin{tabular}{cccc}
\hline Variable & No & Yes & Yes (\%) \\
\hline PTB 1 a & 380,360 & 21,732 & 5.40 \\
PTB 2 & 393,165 & 8927 & 2.22 \\
PTB 3 & 374,340 & 27,752 & 6.90 \\
PTB 4 & 373,247 & 28,845 & 7.17 \\
Benzodiazepine & 165,773 & 236,319 & 58.77 \\
Calcium Channel Blocker & 398,352 & 3740 & 0.93 \\
Diabetes_2002 & 401,226 & 866 & 0.22 \\
Diabetes_2003 & 401,079 & 1013 & 0.25 \\
Diabetes_2004 & 400,833 & 1259 & 0.31 \\
Diabetes_2005 & 400,306 & 1786 & 0.44 \\
Diabetes_2006 & 400,348 & 1744 & 0.43 \\
Diabetes_2007 & 400,302 & 1790 & 0.45 \\
Diabetes_2008 & 400,211 & 1881 & 0.47 \\
\hline
\end{tabular}


Table 1. Cont.

\begin{tabular}{|c|c|c|c|}
\hline Variable & No & Yes & Yes (\%) \\
\hline Diabetes_2009 & 400,062 & 2030 & 0.50 \\
\hline Diabetes_2010 & 399,833 & 2259 & 0.56 \\
\hline Diabetes_2011 & 399,491 & 2601 & 0.65 \\
\hline Diabetes_2012 & 399,027 & 3065 & 0.76 \\
\hline Diabetes_2013 & 398,048 & 4044 & 1.01 \\
\hline Diabetes_2014 & 395,699 & 6393 & 1.59 \\
\hline Depression_2002 & 400,551 & 727 & 0.18 \\
\hline Depression_2003 & 400,328 & 950 & 0.24 \\
\hline Depression_2004 & 400,068 & 1210 & 0.30 \\
\hline Depression_2005 & 399,467 & 1811 & 0.45 \\
\hline Depression_2006 & 399,112 & 2166 & 0.54 \\
\hline Depression_2007 & 398,494 & 2784 & 0.69 \\
\hline Depression_2008 & 398277 & 3001 & 0.75 \\
\hline Depression_2009 & 397,877 & 3401 & 0.85 \\
\hline Depression_2010 & 397,422 & 3856 & 0.96 \\
\hline Depression_2011 & 396,951 & 4327 & 1.08 \\
\hline Depression_2012 & 395,929 & 5349 & 1.33 \\
\hline Depression_2013 & 395,971 & 5307 & 1.32 \\
\hline Depression_2014 & 395,837 & 5441 & 1.36 \\
\hline GERD_200̄2 b & 399,076 & 3016 & 0.75 \\
\hline GERD_2003 & 398,129 & 3963 & 0.99 \\
\hline GERD_2004 & 396,932 & 5160 & 1.28 \\
\hline GERD_2005 & 395,351 & 6741 & 1.68 \\
\hline GERD_2006 & 393,244 & 8848 & 2.20 \\
\hline GERD_2007 & 389,177 & 12,915 & 3.21 \\
\hline GERD_2008 & 386,219 & 15,873 & 3.95 \\
\hline GERD_2009 & 380,452 & 21,640 & 5.38 \\
\hline GERD_2010 & 376,619 & 25,473 & 6.34 \\
\hline GERD_2011 & 372,819 & 29,273 & 7.28 \\
\hline GERD_2012 & 368,833 & 33,259 & 8.27 \\
\hline GERD_2013 & 367,240 & 34,852 & 8.67 \\
\hline GERD_2014 & 363,411 & 38,681 & 9.62 \\
\hline Hypertension_2002 & 401,492 & 600 & 0.15 \\
\hline Hypertension_2003 & 401,464 & 628 & 0.16 \\
\hline Hypertension_2004 & 401,360 & 732 & 0.18 \\
\hline Hypertension_2005 & 401,196 & 896 & 0.22 \\
\hline Hypertension_2006 & 401,088 & 1004 & 0.25 \\
\hline Hypertension_2007 & 400,968 & 1124 & 0.28 \\
\hline Hypertension_2008 & 400,844 & 1248 & 0.31 \\
\hline Hypertension_2009 & 400,718 & 1374 & 0.34 \\
\hline Hypertension_2010 & 400,714 & 1378 & 0.34 \\
\hline Hypertension_2011 & 400,738 & 1354 & 0.34 \\
\hline Hypertension_2012 & 400,406 & 1686 & 0.42 \\
\hline Hypertension_2013 & 400,187 & 1905 & 0.47 \\
\hline Hypertension_2014 & 399,850 & 2242 & 0.56 \\
\hline In Vitro Fertilization & 401,965 & 127 & 0.03 \\
\hline Myoma Uteri & 385,015 & 17,077 & 4.25 \\
\hline Nitrate & 400,776 & 1316 & 0.33 \\
\hline Periodontitis_2002 & 401,895 & 197 & 0.05 \\
\hline Periodontitis_2003 & 401,830 & 262 & 0.07 \\
\hline Periodontitis_2004 & 401,688 & 404 & 0.10 \\
\hline Periodontitis_2005 & 401,665 & 427 & 0.11 \\
\hline Periodontitis_2006 & 401,502 & 590 & 0.15 \\
\hline Periodontitis_2007 & 401,783 & 309 & 0.08 \\
\hline Periodontitis_2008 & 401,795 & 297 & 0.07 \\
\hline Periodontitis_2009 & 401,742 & 350 & 0.09 \\
\hline Periodontitis_2010 & 401,753 & 339 & 0.08 \\
\hline
\end{tabular}


Table 1. Cont.

\begin{tabular}{cccc}
\hline Variable & No & Yes & Yes (\%) \\
\hline Periodontitis_2011 & 401,797 & 295 & 0.07 \\
Periodontitis_2012 & 401,837 & 255 & 0.06 \\
Periodontitis_2013 & 401,824 & 268 & 0.07 \\
Periodontitis_2014 & 401,854 & 238 & 0.06 \\
Prior Cone & 401,911 & 181 & 0.05 \\
Progesterone & 307,684 & 94,408 & 23.48 \\
Proton Pump Inhibitor & 276,084 & 126,008 & 31.34 \\
Region (City) & 28,615 & 373,477 & 92.88 \\
Sleeping Pills & 370,303 & 31,789 & 7.91 \\
Tricyclic Antidepressant & 339,026 & 63,066 & 15.68 \\
\hline
\end{tabular}

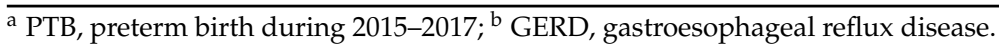

Table 2. Model performance.

\begin{tabular}{ccccccccc}
\hline \multirow{2}{*}{ Model } & \multicolumn{4}{c}{ Accuracy } & \multicolumn{3}{c}{ AUC $^{\mathbf{a}}$} \\
\cline { 2 - 9 } & PTB 1 $^{\mathbf{b}}$ & PTB 2 & PTB 3 & PTB 4 & PTB 1 & PTB 2 & PTB 3 & PTB 4 \\
\hline Logistic Regression & 0.9450 & 0.9766 & 0.9308 & 0.9283 & 0.5536 & 0.5916 & 0.5599 & 0.5610 \\
Artificial Neural & 0.9450 & 0.9766 & 0.9308 & 0.9283 & 0.5000 & 0.5000 & 0.5000 & 0.5000 \\
$\quad$ Network & 0.9450 & 0.9766 & 0.9308 & 0.9283 & 0.5275 & 0.5585 & 0.5407 & 0.5407 \\
Random Forest & &
\end{tabular}

${ }^{a}$ Area under the receiver-operating-characteristic curve; ${ }^{b}$ PTB, preterm birth during 2015-2017.

Table 3. Model performance with undersampling.

\begin{tabular}{ccccccccc}
\hline \multirow{2}{*}{ Model } & \multicolumn{4}{c}{ Accuracy } & \multicolumn{3}{c}{ AUC $^{\mathbf{a}}$} \\
\cline { 2 - 9 } & PTB 1 & PTB 2 & PTB 3 & PTB 4 & PTB 1 & PTB 2 & PTB 3 & PTB 4 \\
\hline Logistic Regression & 0.9448 & 0.9691 & 0.9302 & 0.9276 & 0.5550 & 0.5872 & 0.5567 & 0.5621 \\
Artificial Neural & 0.9450 & 0.9766 & 0.9308 & 0.9283 & 0.5000 & 0.5000 & 0.5000 & 0.5000 \\
$\quad$ Network & 0.9399 & 0.9550 & 0.9251 & 0.9218 & 0.5535 & 0.5803 & 0.5517 & 0.5601 \\
Random Forest & &
\end{tabular}

${ }^{a}$ Area under the receiver-operating-characteristic curve; ${ }^{b}$ PTB, preterm birth during 2015-2017.

Based on random forest variable importance, top-40 determinants of preterm birth during 2015-2017 included socioeconomic status, age, proton pump inhibitor, benzodiazepine, tricyclic antidepressant, sleeping pills, progesterone, GERD for the years 2002-2014, particulate matter for the months January2014-December 2014, region, myoma uteri, diabetes for the years 2013-2014 and depression for the years 2011-2014. These values were the averages for PTB 1, PTB 2, PTB 3 and PTB 4 (Supplementary Figure S1 for each of PTB 1, PTB 2, PTB 3 and PTB 4). The importance rankings of particulate matter were particularly high for PTB 2: PM_2014_08 (5th), PM_2014_12 (6th), PM_2014_02 (7th), PM_2014_11 (8th), PM_2014_09 (10th), PM_2014_06 (11th), PM_2014_10 (12th), PM_2014_01 (13th), PM_2014_07 (14th), PM_2014_05 (15th), PM_2014_03 (17th), PM_2014_04 (18th). These findings were similar with those of undersampling in Supplementary Figure S2. The results of logistic regression (Tables 4 and 5) provide useful information about the sign and magnitude for the effect of a major determinant on preterm birth. For example, the odds of PTB 4 will increase by $12.6 \%$ if socioeconomic status decreases by 10 in Table 4, e.g., from 2 to 12 (median). The odds of PTB 4 will increase by $24.1 \%$ if particulate matter in 2014 August (PM_2014_08) increase by $1 \times 10^{-6} \mathrm{~g} / \mathrm{m}^{3}$ in the table. In a similar vein, the odds of PTB 4 will be greater by $12.2 \%$ for those with depression in 2010 than those without it in the table. 
Table 4. Coefficients of determinants from logistic regression for each type of preterm birth.

\begin{tabular}{|c|c|c|c|c|}
\hline Determinant & PTB $1^{a}$ & PTB 2 & РTB 3 & РTB 4 \\
\hline Age & $* * 1.0000$ & $* * 1.0000$ & 1.0000 & $* * 1.0000$ \\
\hline Benzodiazepine & $* * 1.0004$ & $* * 1.6725$ & $* * 1.0034$ & $* * 1.0017$ \\
\hline $\begin{array}{c}\text { Calcium Channel } \\
\text { Blocker }\end{array}$ & * 1.6383 & 1.5038 & * 1.1644 & 1.0681 \\
\hline Diabetes_2002 & 1.8353 & 2.5656 & 2.3002 & 1.5692 \\
\hline Diabetes_2003 & 1.9481 & 2.0809 & 1.2222 & 1.1303 \\
\hline Diabetes_2004 & 2.4480 & 1.6662 & 1.9147 & 1.7558 \\
\hline Diabetes_2005 & 1.0359 & $* * 1.5251$ & 1.0404 & ** 1.683 \\
\hline Diabetes_2006 & 1.5526 & 1.5065 & 1.2636 & 2.0387 \\
\hline Diabetes_2007 & 1.7720 & 1.1203 & 2.4172 & 1.3727 \\
\hline Diabetes_2008 & 1.9223 & 2.3487 & 1.1073 & 2.5957 \\
\hline Diabetes_2009 & 1.3161 & 2.0164 & 1.0789 & *1.2377 \\
\hline Diabetes_2010 & 1.8100 & 1.2418 & 1.7370 & 1.4228 \\
\hline Diabetes_2011 & 1.2535 & 1.2649 & 2.3813 & 1.4582 \\
\hline Diabetes_2012 & 1.5008 & 1.8574 & 1.4972 & 1.1276 \\
\hline Diabetes_2013 & 2.5368 & 1.5377 & 1.7506 & 2.0177 \\
\hline Diabetes_2014 & $* * 1.0077$ & $* * 1.0000$ & 1.0000 & $* * 1.0000$ \\
\hline Depression_2002 & 1.5841 & 1.0117 & $* * 2.1808$ & 2.2863 \\
\hline Depression_2003 & 1.0729 & $* 1.1167$ & 2.0087 & 1.8181 \\
\hline Depression_2004 & 1.8705 & 1.0978 & *1.8549 & 2.0803 \\
\hline Depression_2005 & 1.1511 & 1.3906 & 2.4433 & 1.5790 \\
\hline Depression_2006 & 1.0631 & * 1.8224 & 1.1180 & 1.4364 \\
\hline Depression_2007 & 1.0956 & * 1.7598 & 1.3947 & 1.8300 \\
\hline Depression_2008 & 2.4007 & 1.3747 & 1.5346 & 2.4277 \\
\hline Depression_2009 & 2.1026 & 2.1431 & 2.5796 & 2.0482 \\
\hline Depression_2010 & 1.0200 & $* * 2.6590$ & 1.0441 & $* * 1.1220$ \\
\hline Depression_2011 & 2.4402 & 2.0030 & 1.9169 & 2.1260 \\
\hline Depression_2012 & 1.7774 & 2.6371 & 1.9263 & 1.3044 \\
\hline Depression_2013 & $* * 1.6356$ & 2.0957 & 1.0422 & 1.0106 \\
\hline Depression_2014 & 1.2603 & 1.6195 & $* * 1.4356$ & 1.2745 \\
\hline GERD_2002 & 1.3563 & 1.9083 & 1.2087 & 1.1254 \\
\hline GERD_2003 & 1.1052 & 2.0605 & 1.0871 & *1.1697 \\
\hline GERD_2004 & 2.2782 & 1.5084 & 1.2115 & 1.2032 \\
\hline GERD_2005 & $* * 1.4589$ & 1.1591 & 1.0435 & $* * 1.0084$ \\
\hline GERD_2006 & 2.3188 & 1.7606 & 2.4122 & 1.6032 \\
\hline GERD_2007 & 1.1257 & 1.9426 & 1.5393 & 1.3863 \\
\hline GERD_2008 & 2.0329 & 2.0811 & 1.2242 & 1.2627 \\
\hline GERD_2009 & 1.0689 & * 1.1254 & 1.5055 & 1.9012 \\
\hline GERD_2010 & 1.1983 & 1.5844 & 1.8026 & 2.5059 \\
\hline GERD_2011 & $* * 1.1285$ & 2.4433 & 1.1078 & 1.0503 \\
\hline GERD_2012 & 1.2868 & 1.0417 & $* * 1.311$ & 1.1167 \\
\hline GERD_2013 & 2.1698 & 1.7186 & 2.5039 & 2.2581 \\
\hline GERD_2014 & 2.0394 & 1.4160 & 1.3279 & 1.5451 \\
\hline Hypertension_2002 & 1.0275 & $* * 1.5738$ & 1.0272 & $* * 1.1747$ \\
\hline Hypertension_2003 & 1.1702 & 1.5249 & 2.4853 & 2.5978 \\
\hline Hypertension_2004 & 1.0521 & $* 2.4381$ & 1.7597 & 1.1924 \\
\hline Hypertension_2005 & 1.7869 & 1.5763 & 1.503 & 2.2755 \\
\hline Hypertension_2006 & $* * 1.0203$ & $* * 2.1423$ & 1.0638 & * 1.0457 \\
\hline Hypertension_2007 & 2.4269 & 1.201 & 2.2096 & 2.3402 \\
\hline Hypertension_2008 & 1.3228 & 1.2716 & 1.2146 & 1.2731 \\
\hline Hypertension_2009 & 1.2009 & 1.6697 & 2.1321 & 1.6487 \\
\hline Hypertension_2010 & 1.0225 & $* * 1.0764$ & * 1.0271 & ** 1.0418 \\
\hline Hypertension_2011 & 1.9046 & 1.2264 & 1.0831 & *2.3515 \\
\hline Hypertension_2012 & 1.2585 & 2.5826 & 2.4341 & 2.2499 \\
\hline Hypertension_2013 & 2.4772 & 1.0218 & $* * 1.7588$ & 1.8997 \\
\hline Hypertension_2014 & $* * 1.3136$ & 1.0142 & $* * 1.0008$ & ** 1.0227 \\
\hline In Vitro Fertilization & $* * 1.3427$ & 1.0005 & $* * 1.0120$ & $* * 1.0002$ \\
\hline Myoma Uteri & $* * 1.0000$ & $* * 1.0000$ & $* * 1.0000$ & $* * 1.0000$ \\
\hline Nitrate & 1.9893 & 1.7917 & 1.9809 & 1.4776 \\
\hline
\end{tabular}


Table 4. Cont.

\begin{tabular}{|c|c|c|c|c|}
\hline Determinant & PTB $1^{a}$ & РTB 2 & РTB 3 & РTB 4 \\
\hline Periodontitis_2002 & 1.9718 & 2.0034 & 1.3465 & 2.0526 \\
\hline Periodontitis_2003 & 1.4198 & 1.7779 & 1.4892 & 1.9032 \\
\hline Periodontitis_2004 & $* * 1.1103$ & 1.8267 & 1.2493 & 1.0187 \\
\hline Periodontitis_2005 & 1.2443 & 1.2035 & 1.6319 & 2.5775 \\
\hline Periodontitis_2006 & $* * 1.3461$ & 1.0005 & $* * 1.4314$ & 1.0181 \\
\hline Periodontitis_2007 & 1.3134 & 1.7419 & 1.6292 & 1.7522 \\
\hline Periodontitis_2008 & 1.8989 & 2.0237 & 2.1047 & 1.7572 \\
\hline Periodontitis_2009 & 1.3065 & 1.6335 & 2.2664 & 2.4738 \\
\hline Periodontitis_2010 & 2.0266 & 2.3908 & 1.0987 & * 2.1828 \\
\hline Periodontitis_2011 & 1.3008 & 1.7355 & 1.6395 & 2.6100 \\
\hline Periodontitis_2012 & 2.4308 & 1.3264 & 1.1636 & 1.7730 \\
\hline Periodontitis_2013 & 1.1598 & 2.5870 & 1.2379 & 1.2664 \\
\hline Periodontitis_2014 & 1.3858 & 1.5461 & 1.5541 & 1.2876 \\
\hline PM_2014_01 & $* * 1.0000$ & $* * 1.0639$ & $* * 1.0001$ & $* * 1.0002$ \\
\hline PM_2014_02 & $* * 1.0000$ & $* * 1.0483$ & ** 1.0000 & ** 1.0000 \\
\hline PM_2014_03 & $* * 1.0214$ & $* * 1.0028$ & $* * 1.0000$ & $* * 1.0003$ \\
\hline PM_2014_04 & 1.9584 & 1.0355 & ** 1.0887 & *1.1566 \\
\hline PM_2014_05 & $* * 1.0032$ & $* * 1.0006$ & $* * 1.0005$ & $* * 1.0004$ \\
\hline PM_2014_06 & ** 1.0103 & $* * 1.0000$ & 1.0008 & ** 1.0000 \\
\hline PM_2014_07 & $* * 1.0000$ & $* * 2.0301$ & 1.0000 & $* * 1.0000$ \\
\hline PM_2014_08 & 2.5322 & 1.2738 & 1.1038 & *1.2412 \\
\hline PM_2014_09 & $* * 1.0001$ & ** 1.1734 & 1.0059 & ** 1.0001 \\
\hline PM_2014_10 & ** 1.0020 & $* * 2.6945$ & 1.0041 & ** 1.0007 \\
\hline PM_2014_11 & 1.7164 & 1.4224 & 1.7286 & 1.2650 \\
\hline PM_2014_12 & $* * 1.0631$ & * 1.5461 & 1.0958 & * 1.0325 \\
\hline Prior Cone & 1.1881 & 1.7899 & 2.5560 & 2.1212 \\
\hline Progesterone & ** 1.0000 & $* * 1.0000$ & $* * 1.0000$ & $* * 1.0000$ \\
\hline Proton Pump Inhibitor & * 1.0314 & $* * 1.8051$ & 1.1315 & 1.0870 \\
\hline Region (City) & * 1.0000 & $* * 1.0068$ & ** 1.3983 & 1.0564 \\
\hline Sleeping Pills & 1.1783 & 1.4780 & 1.6950 & 1.9664 \\
\hline Socioeconomic Status & $* * 1.6547$ & 1.5079 & 1.0856 & *1.0126 \\
\hline Tricyclic Antidepressant & $* * 1.0065$ & $* * 1.3169$ & 1.0613 & * 1.0223 \\
\hline
\end{tabular}

Table 5. Coefficients of determinants from logistic regression with undersampling.

\begin{tabular}{|c|c|c|c|c|}
\hline Determinant & PTB $1^{a}$ & РTB 2 & РTB 3 & РTB 4 \\
\hline Age & $* * 1.0000$ & $* * 1.0000$ & 1.0000 & $* * 1.0000$ \\
\hline Benzodiazepine & $* * 1.0317$ & 1.5222 & ** 1.0198 & 1.3658 \\
\hline $\begin{array}{c}\text { Calcium Channel } \\
\text { Blocker }\end{array}$ & 2.0962 & 1.1379 & 1.6327 & ** 1.0065 \\
\hline Diabetes_2002 & 2.4011 & 1.5789 & 1.4489 & 1.7523 \\
\hline Diabetes_2003 & 1.1577 & ** 1.0046 & 1.6649 & 2.2509 \\
\hline Diabetes_2004 & 1.2701 & 1.4161 & 1.2806 & 2.0063 \\
\hline Diabetes_2005 & ** 1.0306 & 1.6731 & 1.6038 & $* * 1.0338$ \\
\hline Diabetes_2006 & 1.7435 & 2.5349 & 1.8879 & 1.9066 \\
\hline Diabetes_2007 & 2.0364 & 1.5802 & 1.4317 & 2.2514 \\
\hline Diabetes_2008 & 2.5007 & 1.8168 & 2.1137 & 1.3937 \\
\hline Diabetes_2009 & 2.0164 & ** 1.0272 & 2.3441 & * 1.0568 \\
\hline Diabetes_2010 & *1.0748 & 2.0294 & * 1.0843 & 1.9013 \\
\hline Diabetes_2011 & 1.4167 & 2.6692 & 1.3705 & 1.6768 \\
\hline Diabetes_2012 & 2.0074 & 1.4708 & * 1.0748 & 1.4180 \\
\hline Diabetes_2013 & 2.6481 & 1.4699 & 1.3525 & 1.5072 \\
\hline Diabetes_2014 & $* * 1.0004$ & $* * 1.0000$ & $* * 1.0000$ & $* * 1.0000$ \\
\hline Depression_2002 & 1.9737 & 2.2064 & 1.9423 & 2.0489 \\
\hline Depression_2003 & 1.6566 & 1.2687 & 1.506 & 1.2128 \\
\hline Depression_2004 & 1.5461 & 1.4561 & 1.1245 & 2.2313 \\
\hline
\end{tabular}


Table 5. Cont.

\begin{tabular}{|c|c|c|c|c|}
\hline Determinant & PTB $1^{a}$ & PTB 2 & РTВ 3 & РTB 4 \\
\hline Depression_2005 & 2.1117 & 2.2828 & 1.3139 & *1.0695 \\
\hline Depression_2006 & 1.4251 & 1.6441 & 1.6534 & $* * 1.0422$ \\
\hline Depression_2007 & 1.4740 & 2.0573 & $* * 1.0505$ & 1.9812 \\
\hline Depression_2008 & 1.3009 & 1.7328 & 2.2586 & $* * 1.0480$ \\
\hline Depression_2009 & 2.5888 & 2.5528 & 1.3725 & 2.2751 \\
\hline Depression_2010 & 1.2970 & 1.6665 & 1.4457 & * 1.0910 \\
\hline Depression_2011 & 2.5129 & 1.7514 & 1.4860 & 1.5786 \\
\hline Depression_2012 & 1.4842 & 2.0179 & 1.5231 & 1.3482 \\
\hline Depression_2013 & 1.1655 & 1.4877 & $* * 1.0437$ & $* * 1.0402$ \\
\hline Depression_2014 & 1.2937 & 1.1992 & 1.8507 & 1.6490 \\
\hline GERD_2002 & 2.7067 & 2.5489 & 1.2727 & 2.2506 \\
\hline GERD_2003 & 2.0535 & * 1.0868 & $* * 1.0511$ & 1.2279 \\
\hline GERD_2004 & 1.7188 & 1.1785 & 1.6052 & 1.2509 \\
\hline GERD_2005 & 1.4594 & 2.0748 & 1.1597 & $* * 1.0127$ \\
\hline GERD_2006 & 2.3703 & 2.2252 & 1.7161 & 2.4705 \\
\hline GERD_2007 & 1.7442 & 1.3426 & 2.0336 & 2.5701 \\
\hline GERD_2008 & 1.9237 & 1.7541 & 1.6918 & 1.3901 \\
\hline GERD_2009 & 1.2527 & 1.3282 & 1.5109 & 2.5045 \\
\hline GERD_2010 & 2.0095 & 1.7975 & 1.7073 & 1.2688 \\
\hline GERD_2011 & $* * 1.0214$ & 1.3559 & $* * 1.0440$ & 1.4040 \\
\hline GERD_2012 & 2.3677 & 1.4217 & 1.2529 & 1.9972 \\
\hline GERD_2013 & 2.0424 & 1.4997 & 1.5437 & 1.6973 \\
\hline GERD_2014 & 1.2615 & 1.7767 & *1.0782 & 1.2339 \\
\hline Hypertension_2002 & 1.1105 & 1.5397 & *1.0819 & *1.0678 \\
\hline Hypertension_2003 & 1.2966 & 1.2001 & 2.7089 & 1.7429 \\
\hline Hypertension_2004 & $* * 1.0265$ & 1.3399 & 2.4372 & 1.1225 \\
\hline Hypertension_2005 & 1.8251 & 1.3401 & 1.5551 & 2.3208 \\
\hline Hypertension_2006 & * 1.0748 & 1.9067 & 1.3699 & 1.1140 \\
\hline Hypertension_2007 & 2.3224 & 2.6096 & 2.0325 & 1.1384 \\
\hline Hypertension_2008 & 1.1152 & 1.9666 & 1.3094 & 1.6447 \\
\hline Hypertension_2009 & 2.0016 & 2.6371 & 1.3935 & 1.4603 \\
\hline Hypertension_2010 & 1.5264 & 1.7342 & * 1.0928 & $* * 1.0022$ \\
\hline Hypertension_2011 & 1.3179 & 2.2948 & 1.3472 & 2.2883 \\
\hline Hypertension_2012 & 1.4354 & 1.2743 & 1.6905 & 1.6833 \\
\hline Hypertension_2013 & 1.7502 & 1.2018 & * 1.068 & 1.3578 \\
\hline Hypertension_2014 & 1.8333 & $* * 1.0064$ & 1.3089 & 1.7927 \\
\hline In Vitro Fertilization & 1.2642 & 1.1235 & * 1.0986 & 1.7811 \\
\hline Myoma Uteri & $* * 1.0000$ & $* * 1.0000$ & $* * 1.0000$ & $* * 1.0000$ \\
\hline Nitrate & 2.3581 & $* 1.0525$ & 2.1427 & 2.2613 \\
\hline Periodontitis_2002 & 2.2901 & 1.2350 & 1.1919 & 1.5518 \\
\hline Periodontitis_2003 & 2.3085 & 1.1780 & 1.7492 & 1.7112 \\
\hline Periodontitis_2004 & * 1.0720 & 1.1799 & 1.4031 & *1.0831 \\
\hline Periodontitis_2005 & 2.5496 & $* * 1.0224$ & 1.8663 & 1.3206 \\
\hline Periodontitis_2006 & 1.1120 & * 1.0665 & 1.1650 & 1.2683 \\
\hline Periodontitis_2007 & 2.1555 & 1.3009 & 2.3910 & 2.2307 \\
\hline Periodontitis_2008 & 1.7323 & 2.1782 & 1.2830 & 1.4805 \\
\hline Periodontitis_2009 & * 1.0585 & 1.9502 & 1.2883 & 1.1346 \\
\hline Periodontitis_2010 & 2.2747 & 2.5854 & *1.0989 & 1.4304 \\
\hline Periodontitis_2011 & 2.1601 & 1.8406 & 1.6396 & 2.6411 \\
\hline Periodontitis_2012 & 2.4818 & 1.6958 & 2.2394 & 1.3557 \\
\hline Periodontitis_2013 & 1.6737 & 1.5204 & 1.8702 & 1.1657 \\
\hline Periodontitis_2014 & 1.9485 & 2.1991 & 1.2673 & * 1.0691 \\
\hline PM_2014_01 & $* * 1.0001$ & 1.7761 & $* * 1.0000$ & $* * 1.0052$ \\
\hline PM_2014_02 & $* * 1.0000$ & $* * 1.0192$ & $* * 1.0000$ & $* * 1.0000$ \\
\hline PM_2014_03 & 1.1123 & $* * 1.0092$ & $* * 1.0002$ & $* * 1.0000$ \\
\hline PM_2014_04 & 1.5234 & 2.4338 & 1.1769 & 1.3751 \\
\hline PM_2014_05 & $* * 1.0005$ & $* * 1.0099$ & $* * 1.0047$ & $* * 1.0011$ \\
\hline PM_2014_06 & $* * 1.0002$ & $* * 1.0028$ & ** 1.0059 & $* * 1.0002$ \\
\hline
\end{tabular}


Table 5. Cont.

\begin{tabular}{|c|c|c|c|c|}
\hline Determinant & PTB $1^{a}$ & РТВ 2 & РТВ 3 & РТВ 4 \\
\hline PM_2014_07 & $* * 1.0006$ & 2.6328 & ** 1.0013 & ** 1.0003 \\
\hline PM_2014_08 & 1.1148 & * 1.0980 & 2.6242 & 1.3607 \\
\hline PM_2014_09 & $* * 1.0000$ & $* * 1.0053$ & $* * 1.0075$ & $* * 1.0340$ \\
\hline PM_2014_10 & $* * 1.0004$ & * 1.0860 & $* * 1.0042$ & * 1.0966 \\
\hline PM_2014_11 & 1.1574 & 1.8433 & 1.7954 & 1.5836 \\
\hline PM_2014_12 & $* * 1.0367$ & 1.4980 & 1.3242 & 1.2909 \\
\hline Prior Cone & 1.3822 & 2.1422 & 1.3633 & 1.1997 \\
\hline Progesterone & $* * 1.0000$ & $* * 1.0000$ & $* * 1.0000$ & $* * 1.0000$ \\
\hline Proton Pump Inhibitor & ${ }^{*} 1.0945$ & 2.3852 & 1.1117 & 1.1141 \\
\hline Region (City) & $* * 1.0000$ & 1.3816 & 1.2491 & 1.1881 \\
\hline Sleeping Pills & 1.1788 & 1.1526 & 2.6498 & 1.9911 \\
\hline Socioeconomic Status & $* * 1.0314$ & 1.2623 & 1.2843 & ** 1.0041 \\
\hline Tricyclic Antidepressant & $* * 1.0003$ & 2.5491 & 1.3516 & ** 1.0188 \\
\hline
\end{tabular}

a PTB, preterm birth during 2015-2017; ${ }^{*} p<0.10$, ${ }^{* *} p<0.05$.

\section{Discussion}

\subsection{Findings of This Study}

Based on random forest variable importance, top-40 determinants of preterm birth during 2015-2017 included socioeconomic status, age, proton pump inhibitor, benzodiazepine, tricyclic antidepressant, sleeping pills, progesterone, GERD for the years 2002-2014, particulate matter for the months January-December 2014, region, myoma uteri, diabetes for the years 2013-2014 and depression for the years 2011-2014.

\subsection{Summary of Existing Literature}

A recent systematic review reported a positive association between gestational depression and spontaneous preterm labor and birth [6]. This review selected 39 cohort studies with 134,488 participants in total, published in English during 1980-2003. The majority of these studies came from high-income countries such as the United States (27), Denmark (2), France (2), Sweden (2), Canada (1), Norway (1) and the United Kingdom (1). Then, a subsequent systematic review reported that prenatal depression is an important risk factor for preterm birth [7]. This review selected 64 observational studies published in English during 2007 and 2017. Here, 49 (77\%) and $15(23 \%)$ of these studies were done in middle-income and low-income countries, respectively. Likewise, two systematic reviews [8,9] stated a positive relationship between air pollution and preterm birth. These reviews selected 15 articles during 1966-2009 and 14 articles during 1995-2012, respectively. These 27 observational or cohort studies were characterized by varying numbers of participants (3853-3,545,777) and diverse origins, i.e., Australia (1), China (2), Canada (4), the Czech Republic (1), Korea (2), Spain (1), the United Kingdom (2) and the United States (14). Their odds-ratio range was $1.05-1.15$ regarding $\mathrm{PM}_{2.5}$. It would be worthwhile to review several additional population-based cohort studies [10-15] on a positive association between air pollution and preterm birth as well. These studies employed 50,005-1,742,183 participants, covering various areas and periods including San Joaquin Valley (the United States, 2000-2006) [10], Ohio (the United States, 2007-2010) [11], Utah (the United States, 2002-2010) [12], Ontario (Canada, 2005-2012) [13], Wuhan (China, 2011-2013) [14] and Korea (2010-2013) [15]. Their odds-ratio ranges were 1.01-1.57 and 1.04-1.19 regarding $\mathrm{PM}_{10}$ and $\mathrm{PM}_{2.5}$, respectively. However, the number of predictors in the existing literature above has been limited to 14 . Moreover, no effort has been made based on machine learning in this line of research.

\subsection{Contributions of This Study}

This study presents the most comprehensive analysis for the determinants of preterm birth, using a population-based cohort of 405,586 participants and the richest collection of 90 predictors such as demographic/socioeconomic determinants, particulate matter, 
disease information, medication history and obstetric information. Firstly, this study confirms that depression and particulate matter are major predictors of preterm birth (they were the top-40 determinants of preterm birth in this study). Several researchers focus on behavioral, infectious, neuroendocrine and neuroinflammatory mechanisms between depression and preterm birth [19]. Other researchers develop a hypothesis that air pollution causes systemic inflammation, which in turn leads to preterm birth [20]. Little research has been undertaken and more investigation is needed to explore and evaluate various pathways among depression, particulate matter and preterm birth. The findings of this study demonstrate that what is really needed for effective prenatal care is strong intervention for particulate matter together with active counseling and medication for common depressive symptoms (neglected by pregnant women). Secondly, the results of this study agree with those of a previous study with 731 participants on gastroesophageal reflux disease, medication history and preterm birth [18]: The findings of this previous study highlighted the significance of age, socioeconomic status (below high school graduation), progesterone medication history, gastroesophageal reflux disease, region (city) and gestational diabetes mellitus. Above all, to the best of our knowledge, this study is the first attempt to use machine learning and population data to find the main predictors of preterm birth and evaluate its association with depression and particulate matter. This study will be a good starting point in this direction to find main predictors of preterm birth and draw effective implications for its prevention and management.

\subsection{Limitations of This Study}

Firstly, this study did not examine possible mediating effects among variables. Secondly, this study adopted the binary category of preterm birth as no vs. yes (birth between 20 weeks and 0 day and 36 weeks and 6 days of gestation). But preterm birth can have multiple categories and it will be a good topic for future study to compare different predictors for various categories of preterm birth, e.g., extremely preterm (less than 28 (or 24) weeks), very preterm (28-32 (or 24-32) weeks), moderate to late preterm (32-37 weeks) [2]. Thirdly, four categories of preterm birth were defined based on the ICD-10 Code and this could be a source of potential bias. Fourthly, it was not the scope of this study to explore and evaluate various pathways among depression, particulate matter and preterm birth. Little research has been undertaken and more investigations are needed on this topic. Fifthly, uniting various kinds of deep learning approaches for various kinds of preterm birth data would bring new innovations and deeper insights in this line of research. Finally, further investigations of single vs. multiple gestation would deliver more insights and more detailed clinical implications.

\subsection{Conclusions}

Preterm birth has strong associations with depression and particulate matter. What is really needed for effective prenatal care is strong intervention for particulate matters together with active counseling and medication for common depressive symptoms (neglected by pregnant women).

Supplementary Materials: The following are available online at https: / / www.mdpi.com/2075-441 8/11/3/555/s1, Figure S1: Random forest variable importance, Figure S2: Random forest variable importance- - undersampling, Table S1: ICD-10 code for preterm birth, depression, gastroesophageal reflux disease and periodontitis, Table S2: ATC code for medication.

Author Contributions: Conceptualization, K.-S.L., H.Y.K., G.J.C., S.C.H., M.J.O., H.J.K. and K.H.A.; methodology, K.-S.L., H.-I.K. and K.H.A.; software, K.-S.L., H.-I.K. and K.H.A.; validation, K.S.L., H.-I.K. and K.H.A.; formal analysis, K.-S.L., H.-I.K. and K.H.A.; investigation, K.-S.L., H.I.K. and K.H.A.; resources, K.-S.L., H.-I.K. and K.H.A.; data curation, K.-S.L., H.-I.K. and K.H.A.; writing-original draft preparation, K.-S.L., H.Y.K., G.J.C., S.C.H., M.J.O., H.J.K. and K.H.A.; writingreview and editing, K.-S.L., H.Y.K., G.J.C., S.C.H., M.J.O., H.J.K. and K.H.A.; visualization, K.-S.L., H.-I.K. and K.H.A.; supervision, K.-S.L. and K.H.A.; project administration, K.-S.L. and K.H.A.; 
funding acquisition, K.-S.L. and K.H.A. All authors have read and agreed to the published version of the manuscript.

Funding: This work was supported by the Korea University Medical Center (No. K1925051) and the Ministry of Science and ICT of South Korea under the Information Technology Research Center support program supervised by the IITP (Institute for Information and Communications Technology Planning \& Evaluation) (No. IITP-2018-0-01405).

Institutional Review Board Statement: The study was conducted according to the guidelines of the Declaration of Helsinki, and approved by the Institutional Review Board (IRB) of Korea University Anam Hospital on 5 November 2018 (2018AN0365).

Informed Consent Statement: Informed consent was waived by the IRB given that data were deidentified.

Data Availability Statement: The data presented in this study are not publicly available. But the data are available from the corresponding author upon reasonable request and under the permission of Korea National Health Insurance Service.

Conflicts of Interest: The authors declare no conflict of interest.

\section{References}

1. Liu, L.; Oza, S.; Hogan, D.; Chu, Y.; Perin, J.; Zhu, J.; Lawn, J.E.; Cousens, S.; Mathers, C.; Black, R.E. Global, regional, and national causes of under-5 mortality in 2000-15: An updated systematic analysis with implications for the Sustainable Development Goals. Lancet 2016, 388, 3027-3035. [CrossRef]

2. World Health Organization. News: Preterm Birth. Available online: http://www.who.int/news-room/fact-sheets/detail/ preterm-birth (accessed on 1 December 2020).

3. Magro Malosso, E.R.; Saccone, G.; Simonetti, B.; Squillante, M.; Berghella, V. US trends in abortion and preterm birth. J. Matern. Fetal Neonatal Med. 2018, 31, 2463-2467. [CrossRef] [PubMed]

4. Harrison, M.S.; Goldenberg, R.L. Global burden of prematurity. Semin. Fetal Neonatal Med. 2016, 21, 74-79. [CrossRef]

5. Lee, K.S.; Ahn, K.H. Application of artificial intelligence in early diagnosis of spontaneous preterm labor and birth. Diagnostics 2020, 10, 733. [CrossRef]

6. Staneva, A.; Bogossian, F.; Pritchard, M.; Wittkowski, A. The effects of maternal depression, anxiety, and perceived stress during pregnancy on preterm birth: A systematic review. Women Birth 2015, 28, 179-193. [CrossRef] [PubMed]

7. Fekadu Dadi, A.; Miller, E.R.; Mwanri, L. Antenatal depression and its association with adverse birth outcomes in low and middle-income countries: A systematic review and meta-analysis. PLoS ONE 2020, 15, e0227323. [CrossRef] [PubMed]

8. Bosetti, C.; Nieuwenhuijsen, M.J.; Gallus, S.; Cipriani, S.; La Vecchia, C.; Parazzini, F. Ambient particulate matter and preterm birth or birth weight: A review of the literature. Arch. Toxicol. 2010, 84, 447-460. [CrossRef] [PubMed]

9. Li, X.; Huang, S.; Jiao, A.; Yang, X.; Yun, J.; Wang, Y.; Xue, X.; Chu, Y.; Liu, F.; Liu, Y.; et al. Association between ambient fine particulate matter and preterm birth or term low birth weight: An updated systematic review and meta-analysis. Environ. Pollut. 2017, 227, 596-605. [CrossRef] [PubMed]

10. Weber, K.A.; Yang, W.; Lurmann, F.; Hammond, S.K.; Shaw, G.M.; Padula, A.M. Air pollution, maternal hypertensive disorders, and preterm birth. Environ. Epidemiol. 2019, 3, e062. [CrossRef] [PubMed]

11. DeFranco, E.; Moravec, W.; Xu, F.; Hall, E.; Hossain, M.; Haynes, E.N.; Muglia, L.; Chen, A. Exposure to airborne particulate matter during pregnancy is associated with preterm birth: A population-based cohort study. Environ. Health 2016, 15, 6. [CrossRef] [PubMed]

12. Mendola, P.; Nobles, C.; Williams, A.; Sherman, S.; Kanner, J.; Seeni, I.; Grantz, K. Air pollution and preterm birth: Do air pollution changes over time influence risk in consecutive pregnancies among low-risk women? Int. J. Environ. Res. Public Health 2019, 16, 3365. [CrossRef] [PubMed]

13. Lavigne, E.; Yasseen, A.S.; Stieb, D.M.; Hystad, P.; van Donkelaar, A.; Martin, R.V.; Brook, J.R.; Crouse, D.L.; Burnett, R.T.; Chen, H.; et al. Ambient air pollution and adverse birth outcomes: Differences by maternal comorbidities. Environ. Res. 2016, 148, 457-466. [CrossRef] [PubMed]

14. Qian, Z.; Liang, S.; Yang, S.; Trevathan, E.; Huang, Z.; Yang, R.; Wang, J.; Hu, K.; Zhang, Y.; Vaughn, M.; et al. Ambient air pollution and preterm birth: A prospective birth cohort study in Wuhan, China. Int. J. Hyg. Environ. Health 2016, 219, 195-203. [CrossRef] [PubMed]

15. Kim, Y.J.; Song, I.G.; Kim, K.N.; Kim, M.S.; Chung, S.H.; Choi, Y.S.; Bae, C.W. Maternal exposure to particulate matter during pregnancy and adverse birth outcomes in the Republic of Korea. Int. J. Environ. Res. Public Health 2019, 16, 633. [CrossRef] [PubMed]

16. Brown, M.A.; Magee, L.A.; Kenny, L.C.; Karumanchi, S.A.; McCarthy, F.P.; Saito, S.; Hall, D.R.; Warren, C.E.; Adoyi, G.; Ishaku, S.; et al. Hypertensive disorders of pregnancy: ISSHP classification, diagnosis, and management recommendations for international practice. Hypertension 2018, 72, 24-43. [CrossRef] [PubMed] 
17. Air Korea. Air Quality Information. Available online: https://www.airkorea.or.kr/index (accessed on 1 December 2020).

18. Lee, K.S.; Song, I.S.; Kim, E.S.; Ahn, K.H. Determinants of spontaneous preterm labor and birth including gastroesophageal reflux disease and periodontitis. J. Korean Med. Sci. 2020, 35, e105. [CrossRef] [PubMed]

19. Shapiro, G.D.; Fraser, W.D.; Frasch, M.G.; Séguin, J.R. Psychosocial stress in pregnancy and preterm birth: Associations and mechanisms. J. Perinat. Med. 2013, 41, 631-645. [CrossRef] [PubMed]

20. Vadillo-Ortega, F.; Osornio-Vargas, A.; Buxton, M.A.; Sánchez, B.N.; Rojas-Bracho, L.; Viveros-Alcaráz, M.; Castillo-Castrejón, M.; Beltrán-Montoya, J.; Brown, D.G.; O’Neill, M.S. Air pollution, inflammation and preterm birth: A potential mechanistic link. Med. Hypotheses 2014, 82, 219-224. [CrossRef] [PubMed] 